\title{
The essence of the new blood vessels formation process in cancers based on the example of triple-negative breast cancer
}

\author{
Maciej Tomasz Sobieraj ${ }^{1}$, Michał Piotr Budzik², Anna Maria Badowska-Kozakiewicz² \\ 'Public Central Teaching Clinical Hospital, Warszawa, Poland \\ ${ }^{2}$ Department of Biophysics and Human Physiology, Medical University of Warsaw, Poland
}

Triple-negative breast cancer (TNBC) is still one of the most enigmatic breast cancer types and no firm, efficient therapy has been established yet. Regarding its molecular profile and lack of specific receptors, angiogenesis, as an inseparable part of tumor biology, was taken into consideration as one of the leading causes of tumor progression. Taking into account cancerous neovascularization stages and recent scientific researches, it appears probable to understand the process of tumor progression and separate several ways of inhibiting this crucial phenomenon determining neoplasm invasiveness. Combination of traditional therapy and new antiangiogenic agents is a chance for patients to receive treatment as effective as patients with other types of breast cancer or cancer at all. New therapeutic methods may impact the genome level of carcinogenesis. There are many studies aimed at blocking transformed protooncogenes or restore the activity of tumor suppressor genes. Dealing with the process of cancer cells hypoxia and understanding the role of hypoxia inducible factor (HIF) and vascular endothelial growth factor (VEGF) - main angiogenesis inducers - let us introduce proper drugs blocking their activity (e.g. indenopyrazole 21, bevacizumab). Monoclonal antibodies, genetic therapies and newly synthesized drugs replace previously used therapeutic methods as poly (ADP-ribose) polymerase (PARP) inhibitors, mTOR kinase inhibitors, or Ras inhibitors. These new treatment methods include sunitinib, Aurora A kinase/tyrosine kinase inhibitor ENMD-2076, tested due to their multi-target actions, oncolytic viral therapy or small interfering RNA (siRNA). Despite numerous scientific and clinical studies, triple-negative breast cancer remains difficult to treat and is characterized by significantly worse prognosis, statistically shorter five-year survival rate and tendency to relapse.

NOWOTWORY J Oncol 2019; 69, 1: 18-25

Key words: triple-negative breast cancer, angiogenesis, neovascularization, vascular endothelial growth factor, hypoxia, tumor microenvironment

\section{Triple-negative breast cancer characteristics}

Detection rate improvement, inventing and developing new ways of treatment and increasing accessibility belong to the greatest challenges in modern oncology. According to the National Cancer Registry data, breast cancer is most frequent in Polish women population. The same data come from the USA, Canada and Western Europe, where breast cancer comprises about 22\% of female cancers. Most common medical treatment strategies often include making use of presence on the cancer cell surface particular receptors. These are estrogen (ER), progesterone (PR) and human epidermal growth factor type 2 receptors (HER2) (Fig. 1). Lack of these receptors expression is a leading cause of receptor-negative cancers resistance to traditional therapies. Thus considering triple-negative breast cancer (TNBC), it is impossible to apply hormone or HER2 aimed therapies. This implies the necessity to introduce other therapeutic procedures, which may improve TNBC patients' prognosis, what seems to be the most important challenge in TNBC treatment. 


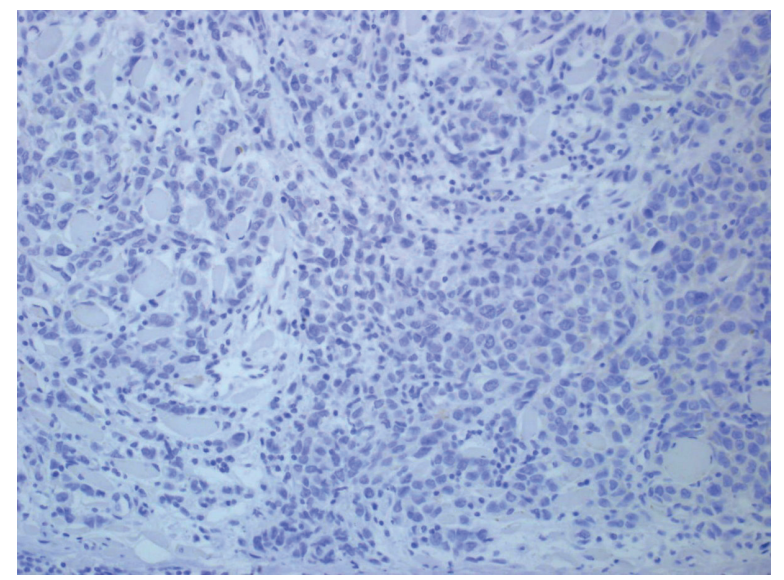

Figure 1. Example of immunohistochemical HER2 staining of triplenegative breast cancer (metaplastic breast cancer), scored 0 (stained cells accounted for less than $10 \%$ of total tumor cells); the micrograph was taken with 20x lens

TNBC is diagnosed in $12-17 \%$ of all breast cancer cases. The risk of this phenotype increases before age of 50 and is connected with BRCA1 mutation more than any other type of breast cancer. As the TNBC cells are frequently relatively heterogeneous and low-differentiated, this cancer type is considered to have an aggressive course, be extremely resistant to chemotherapy, as well as eager to develop some distant metastases and cause short life expectancy thus resulting in extremely poor prognosis [1] (Fig. 2).

The TNBC term is sometimes equated to basal-like breast cancer. This is, though, only partially true, for all oncologists must be aware of distinguishing six subtypes of TNBC: basal-like 1 (BL1), basal-like 2 (BL2), immunomodulatory (IM), mesenchymal (M), mesenchymal stem-like (MSL) and luminal androgen receptor (LAR). However, BL1 is in great majority the kind of basal-like breast cancer sensu stricto (81\%), the rest have its features only in about half of all cases or do not have them at all. In general, 50 to $80 \%$ of TNBCs are also a basal-like breast cancer [2-4].

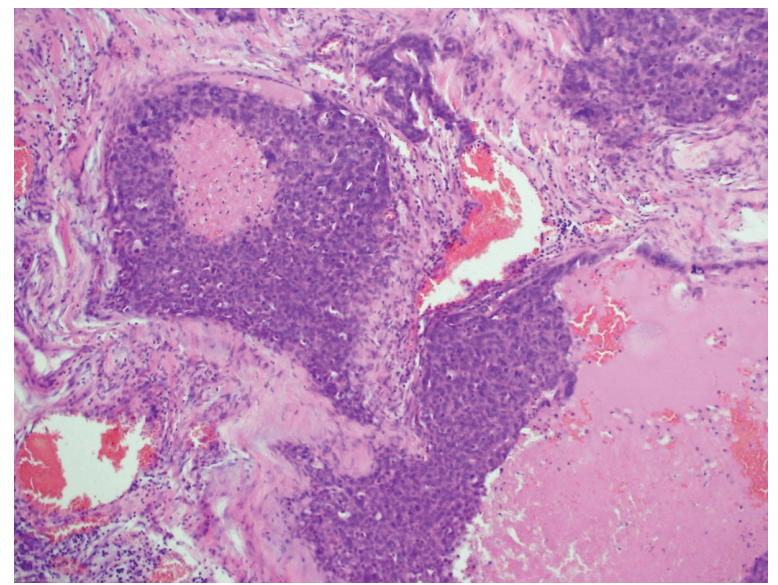

Figure 2. Histopathological image of metaplastic breast cancer assessed as triple-negative breast cancer. Visible necrosis and blood vessels formation, histological grade 3 (G3); original magnification 20x
Basal-like type is characterized not only by lack of ER, PR and HER2 expression, but also a number of features specific for basal cells. These are expressions of: cytokeratin 5, 6, 14, 17, laminin, p53, P-cadherin, common acute lymphoblastic leukemia antigen (CALLA) or smooth muscle actin (SMA), as well as epidermal growth factor receptor (EGFR/HER1) [3, 5-7]. High level of Ki-67 has been recently added to this list as an indicator of high mitotic index and unfavorable prognosis [5].

LAR is the only type of TNBC which could be treated with hormone therapy by using androgen receptor antagonists. Although constituting only about $11 \%$ of TNBCs [3], it cannot be regarded as a proper solution for all patients presented with this type of cancer. It is the other cause forcing scientists to develop non-standard ways of treatment, to which TNBC patients may respond. If we omitted obvious cytostatic therapy and poorly working chemotherapy, there are some therapies under investigation, i.e.: mTOR kinase and PARP1/PARP2 inhibitors or epidermal growth factor receptor (EGFR) and vascular endothelial growth factor (VEGF) inhibitors.

\section{Chemotherapeutic agents used in TNBC therapy}

PARP1 and PARP2 inhibitors (poly (ADP-ribose) polymerase 1 and 2 inhibitors) influence the functioning of poly (ADP-ribose) polymerase and corrupt single-strand damages repair, increasing pace of damages accumulation and as an effect double-strand impairments are very likely to come forth. A very frequent BRCA1 mutation in TNBC patients is worth noticing. This gene is responsible for maintaining double-strand integrality. Its mutation and high mitotic index cause inappropriate functioning of the cell repair mechanisms. Using PARP1 and PARP2 inhibitors may be the cause of cancer cells apoptosis despite their defensive strategies. Iniparib, olaparib and veliparib are used as PARP inhibitors and each of them is currently in stage II of clinical studies as a BL1, BL2 and IM type remedy [8-10].

Mammalian target of rapamycin (mTOR) is a serine threonine kinase which is considered as an intersection of numerous cell metabolic pathways - including growth, survival, and programmed cell death. As its name suggests, rapamycin is a substance, which inhibits the activity of mTOR and rapamycin's derivative - everolimus - is used together with cytostatics in M, MLS and LAR types to prevent distant metastases and to improve the patients' overall prognosis. Despite the low frequency of mTOR/PI3K/AKT signal track genes mutation in TNBC compared to all types of breast cancer, this therapy still significantly increases the response to the treatment also in TNBC patients $[3,11]$.

However, EGFR inhibitors seem to have higher effectiveness in HER2 positive cancers treatment, one of them - gefinitib is also used concerning TNBC, causing cancer cell cycle arrest in approximately $50 \%$ of all cases. The regular treatment includes involving carboplatin and docetaxel together with gefinitib to achieve synergistic reaction [12]. 


\section{Formation of new blood vessels - angiogenesis and neovascularization}

The inevitable part of cancer invasiveness and progression is to strive for maintaining the proper level of cancer cells nourishment. This cannot be achieved without modifying peritumoral vascular environment. The tumor high metabolic rate and constantly increasing cell amount elevate cancerous oxygen and nutrients requirements. Hence the tumors excrete many of proangiogenic and neoangiogenic factors, which promote new vessels formation or bifurcation of already existing ones. This provides a perfect opportunity for invasive, non-adherent cells, to spread into and settle in other organs creating distant metastases.

New blood vessels can be formed in two different ways. The first one is called vasculogenesis and represents developing primitive network of blood vessels from precursor cells named hemangioblasts. These "vascular seeds" are disseminated from blood islands located in yolk sack at the early stages of embryo development. Afterwards the angiogenesis follows, which is much more dynamic process, depending on the body state and controlled by specific substances produced by numerous cells - cancerous or intact ones. It consists in forming new vessels from the endothelium of already existing ones and it is much more frequent phenomenon than vasculogenesis [13]. Angiogenesis is considered as a real breakthrough in tumor progression process. Until the moment when the necessary sources of oxygen and nutrients are provided, even the most malignant cancer is strictly confined and the relative equilibrium concerning cancer cells mitosis and deaths is maintained [14]. As soon as the proangiogenic factors are activated and antiangiogenic are inhibited, a cell or cell group becomes phenotypically proangiogenic, what will be described later on.

Neovascularization or neoangiogenesis is a term thatrefers only to pathological vessels growth, i.e. during tumor development and is described as forming new capillaries in the cancer parenchyma or surrounding, what classifies it as an option of angiogenesis [15]. Neoangiogenesis refers to this phenomenon in neoplasm, since angiogenesis is responsible for the formation of blood vessels in healthy tissue. However, the prefix "neo-" was quite evocative suggesting "new" (gr. veo-: neo-, from véoc: néos - "new, young") and pathological ("neoplasm") matter, it has not fully superseded previous term and coexists worldwide with "angiogenesis" in reference to cancer vessels growth.

Angiogenesis is a complex process, which is crucial for tumor progression. Each of its stages, including endothelial cells activation, endothelium fenestration, intracellular matrix degradation, migration of activated cells and finally forming brand new vessels, one by one require constant stimulation by proangiogenic agents, primarily VEGF, and inhibition of antiangiogenic substances, thrombospondin 1 (TSP-1) above all. Cancer cells mass would be definitely too small to produce such quantities of VEGF as well as RAS mutation, which cause impaired transcription of TSP-1 gene and is frequently present in cancer cells, is not enough to significantly influence the amount of TSP in the tumor surrounding [16].

\section{"Angiogenic field" conception}

Angiogenic field conception was firstly described by Kalas et al. (2005) [17]. It assumes that, together with decreased amount of TSP, cancer cells produce and excrete substances, which lower the expression of TSP-1 gene in non-transformed ambient cells and cause balance shift to the presence of angiogenic actions. Such formation is also called "heterogeneous angiogenic unit". Angiogenic fields cannot be mistaken for hot spots, frequently appearing in the literature $[18,19]$. Hot spots are specific tumor areas, where the density of neovascularizated vessels is much higher. What is more, hot spots presence is connected with poor prognosis and worse response to the chemo- and radiotherapy $[20,21]$. There is currently no scientific evidence if angiogenic fields and hot spots of angiogenesis share the same location during different cancer progression stages.

As the study by Kalas et al. (2005) has revealed, cancer cells do not have to affect angiogenesis process directly. TSP-1 gene decreased expression level in healthy cells can be also modified by presence of thrombocytes and facilitators of inflammation, which are frequently present around the tumor cells [22-24]. This phenomenon perfectly presents angiogenic fields influence on coagulation system.

\section{Angiogenic phenotype and tumor suppressor genes}

Angiogenesis had been considered as a process, which is secondary to the cancerous transformation. Tumor suppressor genes mutations or loss of heterozygosity $(\mathrm{LOH})$ in specific diseases (i.e. Li-Fraumeni syndrome $[25,26]$ ) had not been connected with proangiogenic processes, but only with malignant changes in cells. It was the cancerous tissue growth and its abnormal pace, which was thought to be responsible for switching to proangiogenic phenotype and angiogenesis itself. Further research found that, however, the previous idea was correct and hypoxia does play an enormous role in angiogenesis, cancer cells also have another way to induce this process crucial for their growth. An in vitro fibroblasts culture was organized and they were taken from patient who suffered from Li-Fraumeni syndrome. It transpired that the proangiogenic phenotype, presented as a significant decrease ofTSP-1 gene expression, appeared immediately after performing $\mathrm{LOH}$ and together with cancerous transformation, despite proper oxygenation conditions [27].

\section{Oncogenes as neoplasm's vascular processes modifiers}

Angiogenesis regulation from oncogenes concerns primarily Ras/VEGF relations. Over 20 years ago it become clear, that Ras gene mutation not only leads cells to the cancerous transfor- 
mation, but also induces proangiogenic phenotype [28]. In vitro culture of human intestine epithelium cells with faulted Ras gene began to present proangiogenic features - stimulating endothelial cells growth and their migration. They also started becoming cancerogenic after in vivo implantation. That peculiar feature was absent immediately after VEGF blocking antibodies usage, what confirmed, that Ras gene influences the signal pathway of VEGF production and release. Another oncogenes acting as vascular processes modifiers are: HER2, EGFR, PTTG1 and HPV-16 which stimulate VEGF production. C-myc and Py MT inhibit TSP. Mutated V-Src gene is capable of acting both aforementioned ways (Fig. 1) [16, 29].

\section{Tumor cell hypoxia induces angiogenesis}

A phenomenon of hypoxia is the most important factor inducing vascularization process lifelong. We must not consider hypoxia as an unequivocally negative process. There are number of conditions, when it is positive, even desirable act - e.g. extra capillarization in skeletal muscles as an effect of long-term exercises and repeated hypoxia periods or staying high above sea level, when adaptation to hypoxia includes some changes like capillarization or increasing erythropoietin synthesis and secretion $[29,30]$. At the same time, the awareness of its possible devastating role is absolutely necessary. Anoxic cancer cells produce the same cytokines as healthy anoxic cells would in order to signal to the organism a burning need of more oxygen. This kind of "cells cry for help" is hypoxia-inducible factor (HIF) - the most important but not the only one VEGF activator [31]. HIF is a transcription factor consisting of two heterodomains, a and $\beta$, which are detected in couple of isoforms, e.g. HIF-1 $\beta$ is constantly present in human cells nucleus, HIF-1a and $\mathrm{HIF}$-2a are the proper proangiogenic agents and $\mathrm{HIF-3a}$ is even a particle inhibiting VEGF expression. VEGF expression is elevated because of HIF joining its gene promoter, as well as elimination half-life of VEGF $m R N A$ is lengthened due to binding specific proteins to their $3^{\prime}$ end $[32,33]$. Fledgling vessels are frequently underdeveloped and subnormal, additionally the tumor growth constantly occurs, what leads to deepen hypoxia and the vicious cycle is completed (Fig. 3). HIF neutralization is based on two processes. The first one is oxygen caused HIF hydroxylation and later joining with $\mathrm{vHL}$ protein and ubiquitination. Situation of impaired HIF-hydroxylase enzyme or vHL is identical to hypoxia, considering angiogenesis promotion.

VEGF family consists of VEGF-A, PGF (placental growth factor), VEGF-B, VEGF-C, VEGF-D and VEGF-E. Among them, VEGF-A plays the most significant role. It influences VEGF receptor 1 (VEGFR1) and VEGF receptor 2 (VEGFR2), located only on the surface of endothelial cells and the signal is afterwards conducted through tyrosine kinase cascade. VEGF-C and -D affect endothelium of lymphatic vessels through VEGF receptor 3 (VEGFR3). Vascular endothelial growth factor not only induces cells mitoses and proliferation, but also enables blood serum

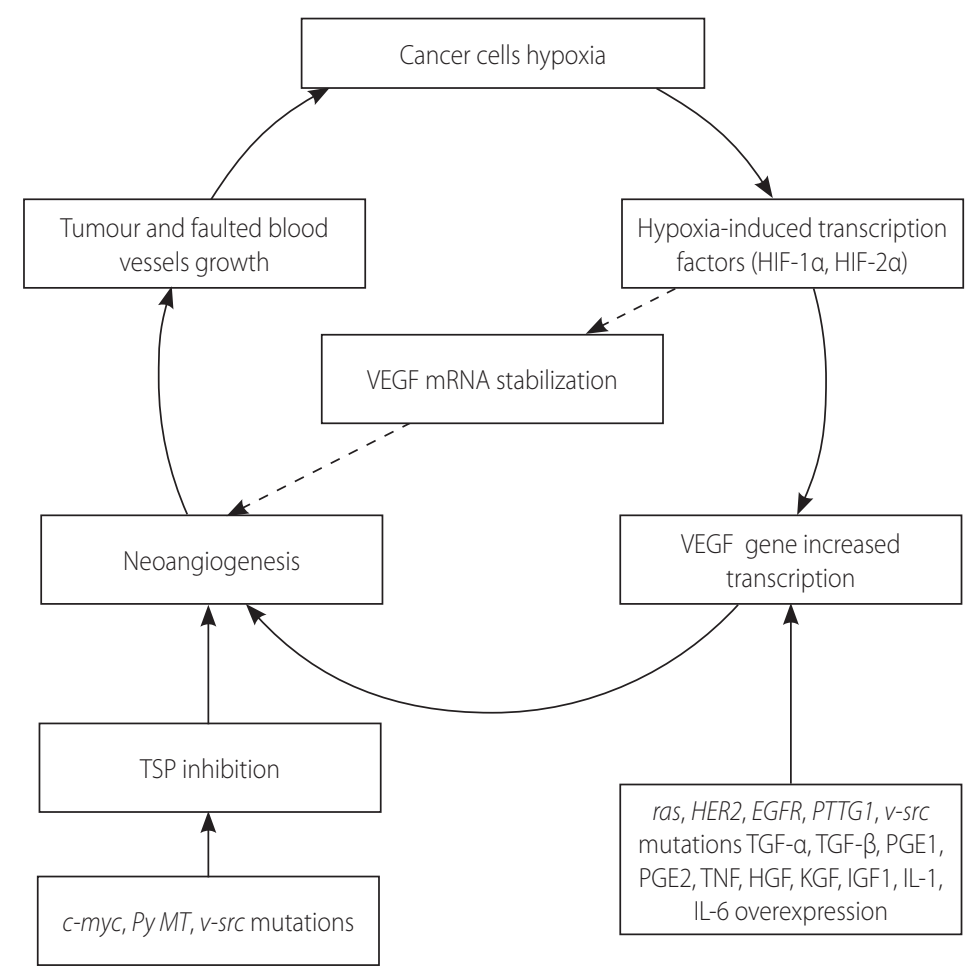

HIF - hypoxia inducible factor; PTTG1 - pituitary tumor-transforming gene 1 (securin); TGF - tumor growth factor; TNF - tumor necrosis factor; HGF - hepatocyte growth factor (scatter factor); KGF - keratinocyte growth factor; IGF - insulin-like growth factor; VEGF - vascular endothelial growth factor; TSP - thrombospondin; Py MT - polyoma middle tumor gene; EGFR - epithelial growth factor receptor; PGE - prostaglandin E; IL - interleukin

Figure 3. Vicious circle of hypoxia-induced neovascularization and other proangiogenic factors influence 
Table I. Endogenous angiogenesis inhibitors

\begin{tabular}{|ll}
\hline Endogenous angiogenesis inhibitor & Source \\
\hline Vascular endothelial growth inhibitor (VEGI) & Endothelium cells \\
\hline Thromospondin-1 (TSP-1) & Platelets, immature astrocytes during brain development \\
\hline Platelet factor 4 (PF4) = chemokine (C-X-C motif) ligand 4 (CXCL4) & Platelets \\
\hline Interleukin 12 (IL-12) & Dendritic cells, macrophages, B-lymphoblastoid cells \\
\hline Angiostatin & Autoproteolytic or enzyme-induced cleavage of plasminogen \\
\hline Angiopoietin 2 & Sites of vascular remodeling \\
\hline Interferon a (IFN-a) & Monocytes, macrophages, "null"cells \\
\hline Interferon $\beta($ IFN- $\beta)$ & Fibro blasts \\
\hline Interferon $\gamma($ IFN- $-\gamma)$ & T-cells, natural killer cells, natural killer T-cells \\
\hline
\end{tabular}

or plasma to extravasate by effectively (50 000 times more) eliciting endothelial pores formation. It allows fibrinogen to transform into fibrin and provides comfortable conditions for cancer cells to organize their new basis, what pays dividends not only in creating suitable stroma, but also separating cancer mass from immunocompetent cells present in or approaching the tumor location. This makes VEGF overexpression serious immunosuppressive factor in pathogenesis of such neoplasms as: breast and stomach cancer, non-Hodgkin lymphomas or small-cell lung carcinoma [13, 34]. Recent researches also reveal that the level of VEGF expression is significantly higher in TNBC than in any other type of breast cancer and may be even a grading factor in differentiation of TNBC from non-TNBC. This border has been set at the level of $665 \mathrm{pg} / \mathrm{ml}$ with expected sensitivity and specificity of $88.9 \%$ and $99.3 \%$ [35].

\section{Angiogenesis and its inhibitors}

Each of aforementioned angiogenesis inducers can be inhibited by proper treatment. The main task of contemporary clinical oncology is to improve results of using medications by defining targeted, associated therapies for all neoplasm subtypes. The process of angiogenesis, which is inevitable in all invasive forms, should be considered as one of the most important research directions. Angiogenesis inhibitors can be divided in two groups.

The first one consists of endogenous inhibitors. Their deactivation, inhibition or lack of expression, is a crucial requirement for cancer to maintain neovascularization, oxygenation and nourishment at the proper level. Among endogenous angiogenesis inhibitors we can distinguish TSP, aforementioned endothelium cells mitosis and differentiation inhibitor, angiostatin, protein constituting from plasminogen after its lysis, immunological cytokines, e.g. interferons and interleukins. What is extremely important for tumor mass, it is an uninterrupted contact with any kind of basis. Stroma remodeling requires specific enzymes, which allow digesting ingredients of extracellular matrix (ECM) (e.g. collagen type IV) and obviously inhibiting them is the way to stymie cancer cells. These factors are four members of tissue inhibitors of metalloproteinase family (TIMP 1, 2, 3 and 4) [36, 37]. There is also vascular endo- thelial growth inhibitor (VEGI), endothelium cells apoptosis regulator, which induces cell suicide most likely via death receptor-3 (DR3). However, the role of VEGl is proven in small number of cases, still hardly known and requires much more studies [38]. Selected endogenous inhibitors are presented in Table I. This group assumes using as a medicine compounds present constantly in human body, but in insufficiency due to disease process. That is a reason why many researches are concentrated around TIMP family (identical actions provides batimastat and marimastat, which unfortunately performed poorly during clinical tests [39]) and TSP (TSP mimicking peptides: ABT-510 and ABT-898 [40]).

Another way, probably more popular and frequently used, is laboratory synthesis of substances which are able to block VEGF-dependent angiogenesis and it is concerning each level ofVEGF overexpression or action. Among these antiangiogenic substances the monoclonal antibodies againstVEGF definitely dominate. Bevacizumab is a recombinant humanized monoclonal antibody, which was the first commercially available angiogenesis inhibitor. It coats VEGF-A particles and forms an inactive complex, which is easily metabolized and eliminated. Soluble receptors for VEGF are also in use. SFIt-1 and sFlk-1 bind to VEGF, competing thereby with VEGF receptors on the surface of endothelial cells, decreasing direct proangiogenic influence. On the other hand, there are specific substances, which act just the opposite way - their conformation considering binding VEGF-VEGFR site is similar to VEGF particle, what allows it to match with superficial receptors and compete with VEGF, causing the effect as above-mentioned soluble receptors in another way - not reducing active VEGF concentration, but saturating its receptors [37].

Answering the question about VEGF inducing agents expression and increasing angiogenesis indirectly is also an answer to the question about present and potential therapies targets. Exemplary drug aim can be HIF - the most common proangiogenic mediator, signaling hypoxia. Ganetespib induces degradation of all particles protected by chaperone proteins HSP90, what includes HIF-1a (but not HIF-2a) and significantly lowers the VEGF level [41]. Another discovered potential therapeutic course considers using 2-methoxyestradiol 
(2ME2), a compound responsible for interphasic microtubules disruption. After positive clinical trials, this drug may become one of the most effective, because it does not only destabilize cell cytoarchitecture and prevent from exponential tumor growth, but also, as the researchers have found, as a result - it downregulates HIF expression and inhibits angiogenesis. These findings not only have shown a new way of cancer treatment, but also demonstrated relations between those two phenomena [42]. Recent studies revealed that Ras pathway inhibition may be an efficient way to deal with angiogenesis as well. Research on mice have proven that application of exogenous a-miR-132 prevents angiogenic sprouting and as a result ocular neovascularization better than any previous VEGF inhibitor [43]. Further tests are necessary in order to check if such therapy has any hope of success in cancer models.

Proangiogenic switch is well known, but there are also some findings available, indicating that this occurrence may be reversible. Using established human liposarcoma xenograft model, Rogers et al. (2014) revealed that a certain, small number of neoplasm cells coming from one stem cell, after a couple of in vivo cycles, formed microscopic, dormant tumors. These studies have shown that angiogenic phenotype is malleable and can be reverted - spontaneously or inducibly, what unquestionably deserves further attention [44].

We must not forget about undesirable or adverse effects of antiangiogenic therapy. There are many studies confirming shortening of overall survival time in process of VEGF inhibitors treatment. In spite of shrinking of primal tumor mass and inhibiting tumor progression due to its antiangiogenic actions, lack of oxygen and growing hypoxia induce epithelial-mesenchymal transition, process of losing polarity and adhesion factors by cancer epithelium cells. This leads to elevated cancerous cells intravasation and distant metastases are more eager to reveal generalized neoplastic disease develops [45]. Another unwanted effect is possibly high risk of hypertension. McIntyre et al. (2014) presented a couple of VEGF inhibitors' mechanisms leading to increased blood pressure. These are: decreasing of nitric oxide metabolites level and prostacyclin signaling, overexpression of endothelin or volume overload due to shortage of fenestration spots in endothelium [46]. VEGFR-aimed therapy can also induce diabetes development, as VEGFR plays a significant role in endothelium of islets of Langerhans maintaining [37].

\section{Antiangiogenic therapy in TNBC - a new insight}

Because of TNBC characteristics, except for $L$ type, hormonal or HER2 aimed therapy is believed to be irrelevant in this type of cancer. Hence antiangiogenic drugs, which are considered as important adjuvant therapy in other cancers, in TNBC are often a real backbone of treatment process together with PARP inhibitors. Scientists dealing with TNBC are especially aware of neovascularization importance and they are those who frequently introduce brand new look on cancerous neovascularization issue.
In addition to all above-mentioned angiogenesis inhibitors, there are a couple of new ideas for disturbing this process, developed in models of TNBC. One of them is Aurora and angiogenic kinase inhibitor ENMD-2076. This substance is responsible for arresting tumor cells in $\mathrm{G} 2$ phase, a moment of mitosis, when serine-threonine kinase Aurora, frequent anticancer therapy target, plays a crucial role and induces caspase-dependent apoptosis. Interestingly, treatment with ENMD-2076 provides better results in PR, ER and HER2 negative cells, what makes it a perfect solution in TNBC. Another advantage of this treatment type is the fact that p53 mutation, frequently present in TNBC, makes cancer cells even more sensitive for its effects. Additionally, ENMD-2076 is an inhibitor of angiogenic tyrosine kinases - especially VEGFR, disturbing proangiogenic signals transducing, what makes it multi-target drug and defines its efficiency [47]

The other studies investigated the influence of sunitinib on basal-like TNBC type. Chinchar et al. (2014) have proven that sunitinib significantly inhibits apoptosis resistance, mitoses, proliferation and invasion in cultured basal-like breast cancer cells, as well as lowers VEGF protein level. However, the sunitinib therapy must be supported by $\gamma$-secretase inhibitor, for sunitinib promotes growth of breast cancer stem cells (CSCs) through interfering with Notch protein expression and inducing hypoxia. This innovative combined therapy might simultaneously target neovascularization and inhibit breast CSCs mitoses [48]. Aforementioned treatment including ganetespib blocking HIF-1 was also performed successfully on TNBC model [42].

Most recent studies constitute three major milestones in cancer therapy: cycle checkpoint inhibitors, siRNA encapsulated in immunoliposomes and oncolytic virus therapy. All of them were tested on TNBC and last two promise new directions in antiangiogenic therapy. siRNA (small interfering RNA) is a short, double stranded RNA particle, interfering with homologous mRNA and inhibiting transcription. Required precision in affecting only faulted cells and avoiding endogenous enzymes are nowadays ensured by encapsulating with liposomes - immunoneutral carriers. The drug infiltrates tumor mass thanks to enhanced permeability and retention effect and lipid integument is projected to destabilize with inner (decreased pH in tumor environment, elevated temperature) or outer (radiation, ultrasound) factors [49]. Specific siRNA sections have already been tested in vitro $(A M L$ - target: CD33, HER2-positive breast cancer - target: HER2) and in vivo (neuroblastoma - target: disialogangloside GD2). As to TNBC, lipocalin 2 ( $\operatorname{LCn} 2$ ) has been shown to promote epithelial to mesenchymal switch and induce neovascularization process in cancer through VEGF concentration increase. Guo et al. (2016) revealed that intracellular adhesion molecule 1 (ICAM-1) - targeted Lcn2 siRNA-encapsulating liposomes inhibit neovascularization and may be useful in future clinical approach [50].

Natural or genetically engineered viruses infecting and breaking down cancer cells become experimental oncologists'con- 
cern bringing to life the idea of oncolytic virus therapy. Already in 1912 De Pace reported cervical cancer regression case after vaccination against rabies but only after over one hundred years in 2015 first oncolytic virus drug was approved in Europe and USA T-VEC (talimogene laherparepvec), modified herpes simplex virus type 1 introduced to therapy of advanced malignant melanoma, suppressing tumor expansion and expanding overall survival. Viruses in cancer treatment found their places also in hepatocellular carcinoma (vaccinia virus), urinary bladder cancer (adenovirus CG0070) and head and neck cancers (one of reoviruses) [51]. New scientific impact resulted in Gholami et al. (2014) study. Modified vaccinia virus (GLV-1h164) turned out to decrease VEGF level, encoding single chained antibody against this proangiogenic agent. Together with determination of cytotoxicity at the level of 90\% within 4 days, GLV-1 h164 and its potent successors appeared to be a remarkable direction in TNBC therapy [52].

Other pathways suspected to inhibit neovascularization are constantly under investigation and Liang et al. (2017) discovered that activation of G protein-coupled estrogen receptor (GPER) reduced IL-6 level limiting inflammation and VEGF level, corrupting cancerous vessels growth process [53]. Da Motta et al. (2017) described the process of epigenetic affecting of tumor progression and hypoxia. One of bromodomain and extra-terminal inhibitors (BETi) - JQ1, doubly influences cancer growth. Not only does it decrease the amount of hypoxia-induced genes, i.e.VEGF, HIF, but also stops HIF from binding with effectors of neovascularization. Considering TNBC's high aggressiveness, therapy resistance and usually poor outcome, presenting such a pleiotropic characteristic is undoubtedly desirable and promising [54].

The uniqueness of TNBC among other breast cancers was well demonstrated by Wang et al. (2018). Not only is TNBC resistant to common antiestrogen therapy due to lack of hormone receptors, but he also found malignant cells to be vulnerable to just the opposite - estrogen-based therapy. GPER-1 (G-protein-coupled estrogen receptor-1) expression was analyzed in the TNBC cell lines MDA-MB-468 and MDA-MB-436 to be activated by $17 \beta$-estradiol and inhibits VEGF expression disturbing neovascularization, tumor growth and metastatic processes. In vivo trials in mice revealed significantly lower microvascular density (MVD) and tumor size in individuals exposed to $17 \beta$-estradiol than in control group what constitutes estrogen-GPER-1 pathway to be a promising a new TNBC, and cancer in general, treatment method after further investigation and clinical trials [55].

Ribatti et al. (2016) performed a reliable summary of issues concerning angiogenesis phenomenon in TNBC putting emphasis on some specific aspects of the process and different ways of strangling it as well as an objective assessment of clinical outcomes. Presented review clarifies MVD as a significant disease intensity indicator as well as clinical outcome prognostic factor that could be measured and used to compare effectiveness of various therapies. The authors also try to identify precise source of elevated proangiogenic factors in the tumor environment and to present studies convincing them to be tryptase-positive mast cells, which incidence in tumor samples increases proportionally to angiogenesis severity - MVD and presence of micrometastases in sentinel lymph nodes. In spite of decades of auspicious in vitro studies, TNBC is still connected with poor clinical outcome. However, the combination of conventional chemotherapy and antiangiogenic drugs has reached a moderate success and TNBC patients can actually benefit from it, further research is continuously required to fulfill great hopes placed in it. Ribatti et al. (2016) propose to search for more effective therapy in the current of personalized medicine. Targeted therapy achieved with thorough marker diagnostic should allow to project individual scheme for each patient and result in increased survival rate, still the costs of such intention are the most critical concern [56].

\section{Summary}

Process of angiogenesis in TNBC is crucial. The first reason is the same as considering other cancer types - neovascularization must be induced by transformed cells, in other option tumor growth will be limited and cancer will not reveal its potential. The other ground is connected with molecular profile of TNBC cells. Shortage of possible targets for therapy forces scientist and clinical oncologist to seek for solutions in angiogenesis inhibitors more often than in any other cancer type. The will of finding proper TNBC therapy is definitely the main driving force of antiangiogenic factors researches. Numerous new ideas come up every year, but the clinical results most often do not correspond with experimental ones, regarding the use of mice models or trials of inhibiting angiogenesis of human tumors inside animals' body. However, the phenomenon of angiogenesis is still reasonably considered as one of the most important testing direction in TNBC therapy.

Conflict of interests: none declared

\section{Michał Piotr Budzik \\ Medical University of Warsaw \\ Department of Biophysics and Human Physiology \\ ul. Chałubińskiego 5 \\ 02-004 Warszawa, Poland \\ e-mail:mbudzik@wum.edu.pl}

Received: 24 Feb 2019

Aproved to printing: 9 May 2019

\section{References}

1. Dent $\mathrm{R}$, Trudeau M, Pritchard KI et al. Triple-negative breast cancer: clinical features and patterns of recurrence. Clin Cancer Res 2007; 13: 4429-4434.

2. Lehmann BD, Bauer JA, Chen X et al. Identification of human triple-negative breast cancer subtypes and preclinical models for selection of targeted therapies. J Clin Invest 2011; 121: 2750-2767.

3. Nowacka-Zawisza M, Krajewska WM. Triple-negative breast cancer: molecular characteristics and potential therapeutic approaches. Postepy Hig Med Dosw 2013; 67: 1090-1097.

4. Foulkes WD, Smith IE, Reis-Filho JS. Triple-negative breast cancer. NEngl J Med 2010; 363: 1938-1948. 
5. Miyoshi Y, Murase K, Oh K. Basal-like subtype and BRCA1 dysfunction in breast cancers. Int J Clin Oncol 2008; 13: 395-400.

6. Voduc KD, Cheang MC, Tyldesley $S$ et al. Breast cancer subtypes and the risk of local and regional relapse. J Clin Oncol 2010; 28: 1684-1691.

7. Kordek R, Bednarek AK. DNA microarray studies on breast cancer. Onkol Prakt Klin 2005; 1: 10-17.

8. Kozioł M, Püsküllüoglu $M$, Zygulska A. PARP inhibitors and their role in the therapy of triple-negative metastatic breast cancer. Przegl Lek 2012; 69: 265-270.

9. Yelamos J, Farres J, Llacuna L et al. PARP1 and PARP2: New players in tumour development. Am J Cancer Res 2011; 1: 328.

10. Dębska S, Kubicka J, Czyżkowski R et al. PARP inhibitors - theoretical basis and clinical application. Postepy Hig Med Dosw 2012;66: 311-321.

11. Vinayak S, Carlson RW. mTOR inhibitors in the treatment of breast cancer. Oncology (Williston Park) 2013; 27: 38-44.

12. Corkery B, Crown J, Clynes $M$ et al. Epidermal growth factor receptor as a potential therapeutic target in triple-negative breast cancer. Ann Oncol 2009; 20: 862-867.

13. Swidzińska E, NaumnikW, Chyczewska E. Angiogenesis and neoangiogenesis - the role in lung cancer and other tumors. Pneumonol Alergol Pol 2006; 74: 414-420.

14. Rak J, Yu JL, Kerbel RS, Coomber BL. What do oncogenic mutations have to do with angiogenesis/vascular dependence of tumors. Cancer Res 2002; 62: 1931-1934.

15. Napoli C, Giordano A, Casamassimi A et al. Directed in vivo angiogenesis assay and the study of systemic neoangiogenesis in cancer. Int $J$ Cancer 2011; 128: 1505-1508.

16. Rak J. Oncogenes as modifiers of vascular processes associated with cancer. Nowotwory J Oncol 2006; 56: 57-79.

17. Kalas W, Yu JL, Milsom C et al. Oncogenes and angiogenesis. Downregulation of thrombospondin 1 innormal fibroblasts exposed to factors from cancer cells harboring mutant ras. Cancer Res 2005; 65: 8878-8886.

18. Hansen S, Grabau DA, Rose $C$ et al. Angiogenesis in breast cancer: a comparative study of the observer variability of methods for determining microvessel density. Lab Invest 1998; 78: 1563-1573.

19. Linder S, Blåsjö $M$, von Rosen $A$ et al. Pattern of distribution and prognostic value of angiogenesis in pancreatic duct carcinoma: a semiquantitative immunohistochemical study of 45 patients. Pancreas 2001; 22: 240-247.

20. Bosari $\mathrm{S}$, Lee AK, DeLellis RA et al. Microvessel quantitation and prognosis in invasive breast carcinoma. Hum Pathol 1992; 23: 755-761.

21. de Jong JS, van Diest PJ, Baak JP. Hot spot microvessel density and the mitotic activity index are strong additional prognostic indicators in invasive breast cancer. Histopathology 2000; 36: 306-312.

22. Kalas W, Klement $\mathrm{P}$, Rak J. Downregulation of the angiogenesis inhibitor thrombospondin 1 in fibroblasts exposed to platelets and their related phospholipids. Biochem Biophys Res Commun 2005; 334: 549-554.

23. Jurasz P, Alonso-Escolano D, Radomski MW. Platelet-cancer interactions: mechanisms and pharmacology of tumour cell-induced platelet aggregation. Brit J Pharmacol 2004; 143: 819-826.

24. Balkwill F, Mantovani A. Inflammation and cancer: back to Virchow? Lancet 2001; 357: 539-545.

25. Malkin D, Li FP, Strong LC et al. Germ line p53 mutations in a familial syndrome of breast cancer, sarcomas, and other neoplasms. Science 1990; 250: 1233-1238.

26. Srivastava S, Zou Z, Pirollo K et al. Germ-line transmission of a mutated p53 gene in a cancer-prone family with Li-Fraumeni syndrome. Nature 1990; 348: 747-749.

27. Rastinejad F, Polverini PJ, Bouck N. Regulation of the activity of a new inhibitor of angiogenesis by a cancer suppressor gene. Cell 1989; 56: 345-355.

28. Rak J, Mitsuhashi $Y$, Bayko $L$ et al. Mutant ras oncogenes upregulate VEGF/VPF expression: implications for induction and inhibition of tumor angiogenesis. Cancer Res 1995; 55: 4575-4580.

29. Sadłecki $P$, Walentowicz-Sadłecka M, Grabiec M. Angiogenesis in neoplastic processes. Przeglad Menopauzalny 2010; 1: 28-31.

30. Breen $\mathrm{E}$, Tang K, Olfert M et al. Skeletal muscle capillarity during hypoxia: VEGF and its activation. High Alt Med Biol 2008; 9: 158-166.

31. Prior SJ, Blumenthal JB, Katzel LI et al. Increased skeletal muscle capillarization after aerobic exercise training and weight loss improves insulin sensitivity in adults with IGT. Diabetes Care 2014; 37: 1469-1475.

32. Arany Z, Foo SY, Ma Y et al. HIF-independent regulation of VEGF and angiogenesis by the transcriptional coactivator PGC-1 $\alpha$. Nature 2008; 451: 1008-1012.
33. Pugh CW, Ratcliffe PJ. Regulation of angiogenesis by hypoxia: role of the HIF system. Nat Med 2003; 9: 677-684.

34. Szala S. Angiogenesis and immune supression: yin and yang of tumor progression? Postepy Hig Med Dosw 2009; 63: 598-612.

35. El-Hefny MA, Afandiyev A, Karimova $S$ et al. Evaluation of vascular endothelial growth factor (VEGF) levels and survival among triple-negative breast cancer (TNBC) and non-TNBC cases. Intern J Adv Sc Tech Res 2013; 3: 22-36.

36. Jarosz P, Woźniak B. Angiogenesis in cancer diseases. Prz Med Uniw Rzesz Inst Leków 2012; 4: 498-507.

37. Stetler-Stevenson WG, Krutzsch HC, Liotta LA. Tissue inhibitor of metalloproteinase (TIMP-2). A new member of the metalloproteinase inhibitor family. J Biol Chem 1989; 264: 17374-17378.

38. Jia W, Sander AJ, Jia G et al. Vascular endothelial growth inhibitor (VEGI) is an independent indicator for invasion in human pituitary adenomas. Anticancer Res 2013; 33: 3815-3822.

39. Sparano JA, Bernarndo P, Stephenson P et al. Randomized phase III trial of marimastat versus placebo in patients with metastatic breast cancer who have responding or stable disease after first-line chemotherapy: eastern cooperative oncology group trial E2196. J Clin Oncol 2004; 22: 4683-4690.

40. Garside SA, Henkin J, Morris KD et al. A Thrombospondin-Mimetic Peptide, ABT-898, Suppresses Angiogenesis and Promotes Follicular Atresia in Pre- and Early-Antral Follicles in Vivo. Endocrinology 2010; 151: 5905-5915.

41. Xiang L, Gilkes DM, Chaturvedi P et al. Ganetespib blocks HIF-1 activity and inhibits tumor growth, vascularization, stem cell maintenance, invasion, and metastasis in orthotopic mouse models of triple-negative breast cancer. J Mol Med (Berl) 2014; 92: 151-164.

42. Mabjeesh NJ, Escuin D, LaVallee TM et al. 2ME2 inhibits tumor growth and angiogenesis by disrupting microtubules and dysregulating HIF. Cancer Cell 2003; 3: 363-375.

43. Westenskow PD, Kurihara T, Aguilar E et al. Ras pathway inhibition prevents neovascularization by repressing endothelial cell sprouting. J Clin Invest 2013; 123: 4900-4908.

44. Rogers MS, Novak K, Zurakowski D et al. Spontaneous reversion of the angiogenic phenotype to a nonangiogenic and dormant state in human tumors. Mol Cancer Res 2014; 12: 754-764.

45. Loges $\mathrm{S}$, Mazzone $\mathrm{M}$, Hohensinner $\mathrm{P}$ et al. Silencing or fueling metastasis with VEGF inhibitors: antiangiogenesis revisited. Cancer Cell 2009; 15: 167-170.

46. McIntyre WF, Oqab Z, Hopman W et al. Hypertension due to antiangiogenic cancer therapy with VEGF inhibitors: is autonomic nervous system toxicity another possible mechanism? Can J Cardiol 2014; 30: 534-543.

47. Diamond JR, Eckhardt SG, Tan AC et al. Predictive biomarkers of sensitivity to the aurora and angiogenic kinase inhibitor ENMD-2076 in preclinical breast cancer models. Clin Cancer Res 2013; 19: 291-303.

48. Chinchar E, Makey KL, Gibson J et al. Sunitinib significantly suppresses the proliferation, migration, apoptosis resistance, tumor angiogenesis and growth of triple-negative breast cancers but increases breast cancer stem cells. Vasc Cell 2014; 6: 12.

49. Meissner JM, Toporkiewicz M, Matusiewicz L et al. Liposomy jako niewirusowe systemy dostarczania leków genetycznych. Postepy Hig Med Dosw 2016; 70: 200-209.

50. Guo P, Yang J, Jia D et al. ICAM-1-targeted, Len2 siRNA-encapsulating liposomes are potent anti-angiogenic agents for triple negative breast cancer. Theranostics 2016; 6: 1-13.

51. Fukuhara $\mathrm{H}$, Ino $\mathrm{Y}$, Todo $\mathrm{T}$. Oncolytic virus therapy: A new era of cancer treatment at dawn. Cancer Sci 2016; 107: 1373-1379.

52. Gholami S, Marano A, Chen NG et al. A novel vaccinia virus with dual oncolytic and anti-angiogenic therapeutic effects against triple-negative breast cancer. Breast Cancer Res Treat 2014; 148: 489-499.

53. Liang $S$, Cheng Z, Jiang $G$ et al. Activation of GPER suppresses migration and angiogenesis of triple negative breast cancer via inhibition of NFKB/IL-6 signals. Cancer Lett 2017; 386: 12-23.

54. da Motta LL, Ledaki I, Purshouse Ket al. The BET inhibitor JQ1 selectively impairs tumour response to hypoxia and downregulates CA9 and angiogenesis in triple negative breast cancer. Oncogene 2017; 36: 122-132.

55. Wang C, Li J, Ye S et al. Oestrogen inhibits VEGF expression and angiogenesis in triple-negative breast cancer by activating GPER-1.J Cancer 2018; 9: 3802-3811.

56. Ribatti D, Nico B, Ruggieri S et al. Angiogenesis and antiangiogenesis in triple-negative breast cancer. Trans/ Oncol 2016; 9: 453-457. 